\title{
Agglomeration Mechanisms and Kinetics during the Carbonation of a Suspension of Lime in a Pilot Batch Reactor
}

\author{
Mathilde Schnebelen ${ }^{1,2^{*}}$, Kevin Mozet ${ }^{1}$, Alexandra Jakob ${ }^{2}$, Didier Sy², Edouard Plasari1, \\ Hervé Muhr ${ }^{1}$ \\ ${ }^{1}$ Université de Lorraine, Laboratoire Réactions et Génie des Procédés, Nancy, France \\ ${ }^{2}$ Solvay Spécialités France, Salin de Giraud, France \\ Email: ${ }^{*}$ mathilde.schnebelen@univ-lorraine.fr
}

Received 10 June 2015; accepted 24 July 2015; published 27 July 2015

Copyright @ 2015 by authors and Scientific Research Publishing Inc.

This work is licensed under the Creative Commons Attribution International License (CC BY). http://creativecommons.org/licenses/by/4.0/

(c) (i) Open Access

\section{Abstract}

The reaction studied in this work is the synthesis of nanometric size calcium carbonate particles by carbonation of a suspension of lime, which represents the most common industrial route. It consists in bubbling carbon dioxide in a suspension of lime to obtain precipitated calcium carbonate (PCC). PCC is a mineral filler with various applications: sealants, paints, paper, ink, pharmacy, cosmetics, food etc. However, there is a challenge related to the synthesis and the use of this precipitate: the agglomeration of the monoparticles. The aim of this work is then to understand the mechanisms of this phenomenon and to study its kinetics to improve the run of the process and the control of its impact on the final product. Experiments realized with a high concentration in sodium chloride $(2 \mathrm{M})$ showed that the modification of the electrostatic environment did not change the particle size distribution and the morphology of the agglomerates. This indicates that the electrostatic interactions are not responsible for the agglomeration but the formation of crystalline bridges induced by the crystal growth. Thus, thanks to an agglomeration model including the crystal growth rate, the agglomeration kernel $\beta$ and the agglomeration constant $\beta_{0}$ can be determined using a mathematical treatment of the experimental particle size distributions. Finally, by varying the experimental conditions, it appears that the agglomeration constant increases with the temperature whereas there is an optimal value regarding the shear rate.

\section{Keywords}

Calcium Carbonate, Agglomeration Kernel, Lime Carbonation, Precipitation

\footnotetext{
${ }^{*}$ Corresponding author.
} 


\section{Introduction}

The reaction of carbonation is the most common industrial route to synthetize precipitated calcium carbonate (PCC). The process to get particles of PCC consists in bubbling a reactive gas (carbon dioxide) in a suspension of calcium hydroxide (also called suspension of lime). The solid compound obtained is a mineral filler, which has applications in various domains such as paints, paper, pharmacy or food.

However, there is a challenge related to the synthesis and the use of this precipitate: the agglomeration of the monoparticles. Indeed, they are agglomerated in micrometric "loose" structures. This agglomeration has consequences on the run of the process and the properties of the final product so the aim of this work is to understand the mechanisms of this phenomenon and to study its kinetics under complex industrial conditions characterized by a high concentration in solid phase and intensive mechanical stirring and gas bubbling.

To answer these questions, a pilot installation has been developed, which is a scale down of the industrial reactor including all the equipment and instrumentation necessary to monitor the different operating parameters of the process. Furthermore, samples are collected all along the reaction in order to follow the evolution of the PCC particle size distribution.

During the experiments, two agglomeration periods are observed. At the beginning of the first period, amorphous calcium carbonate is produced, which is verified by the in situ Raman spectroscopy monitoring. The amorphous calcium carbonate serves as a binding compound for calcium hydroxide particles in suspension creating agglomerates according to a gelling mechanism. During this short period (less than $5 \%$ of the experimental reaction time) the suspension behaves like an extremely viscous but unstable gel, where no any information can be obtained. The amorphous calcium carbonate is rapidly transformed to calcite, so the gel is quickly destroyed and the second period begins. The second period is the principal period of the calcite precipitation characterized by the nucleation, crystal growth and agglomeration of this compound. The nucleation and crystal growth kinetics are presented elsewhere [1], while the aim of this work is to clarify the mechanism and to quantify the kernel expression during the agglomeration of calcite particles. Indeed, this phenomenon sensibly influences the end use properties of the PCC.

The agglomeration is the mechanism through which solid particles collide and stick together by the formation of physical bond called "crystalline bridges". This mechanism can be divided in three steps:

- approach of the particles due to the Brownian movement or the reactor hydrodynamics;

- collision of the particles thanks to electrostatic forces;

- building of a crystalline bridge induced by crystal growth.

The agglomeration rate is often described thanks to a parameter named agglomeration kernel $\beta$ that includes a constant $\beta_{0}$ which describes the tendency of a substance to agglomerate and takes into account the characteristics of the substance and the environment in which it evolves.

There are several methods to determine the agglomeration kernel and they are often divided in four categories:

- methods allowing the simultaneous determination of the agglomeration kernel and the kinetics of nucleation and growth rates ([2]-[9]);

- methods allowing the determination of the agglomeration kernel provided that the nucleation and crystal growth rates are known ([10]-[13]);

- methods allowing the determination of the agglomeration kernel and crystal growth rates in absence of nucleation ([14]-[17]);

- the methods allowing the determination of the agglomeration kernel in a system only under agglomeration ([18] [19]).

All this methods are based on experimental particle size distributions and the solving of a population balance. The methods 3 and 4 are inapplicable in our work, while the methods 1 are applicable but demand the simultaneous adjusting of numerous parameters which is complicated and time consuming. That is why the option chosen for this work belongs to the second category.

Once the agglomeration kernel and more precisely the agglomeration constant $\beta_{0}$ is determined, it is also important to study the impact of the different process parameters on it. Thus carbonations under various temperatures and shear rates (thanks to different stirrer speeds and gas flow rates) were lead to quantify the influence of these factors on this constant. The first author to study the influence of shear rate on the agglomeration was [20] when he expressed the agglomeration kernel as a function of the shear rate. Since then, other authors have pub- 
lished on this subject ([15] [21]-[24]) and have pintpointed for various systems a strong dependence of the agglomeration kernel on the shear rate.

Regarding the temperature, we could cite [25] or [11] that worked on silica and neodymium oxalate respectively and observed an increase of the agglomeration kernel with temperature.

\section{Experimental}

\subsection{Experimental Set-Up}

The experimental pilot scale installation (Figure 1 and Figure 2) is composed of a six liters glass jacketed batch reactor and all necessary equipment and instruments to follow the reaction parameters (temperatures $\mathrm{T}$, $\mathrm{pH}$, gas flowrate $\mathrm{Q}_{\mathrm{m}}$ and conductivity of the suspension $\chi$ ). The $\mathrm{pH}$ sensor used in this work is an industrial model InPro 4800i SG (reference source Ag/AgCl) provided by Mettler Toledo. It is combined with a temperature sensor Platinum 1000 to compensate the modification of temperature. The sensor is calibrated before each experiment thanks to two buffer solutions (pH 7.00 and 10.00 furnished by Fluka) and with the help of the software I SensLight and a pH transmitter M700 both from Mettler Toledo. The reactor is provided in gas thanks to a bottle of $\mathrm{CO}_{2}$ and the compressed air system. The unit also includes two thermo-regulated baths: the first one is settled to $25^{\circ} \mathrm{C}$ in order to regulate the gas temperature which is cooled down earlier due to the gas expansion at the exit of the bottle. The second one allows to adjust the temperature of the double jacket to the desired temperature regarding the suspension of lime. The reactive gas is composed of $30 \% \mathrm{CO}_{2}$ and the mass flow rate is controlled by two mass flowmeters.

\subsection{Experimental Operations}

The reactor is filled with four liters of previously prepared suspension containing $150 \mathrm{~g} \cdot \mathrm{L}^{-1}$ of calcium hydroxide $\left(2.03 \mathrm{~mol} \cdot \mathrm{L}^{-1}\right)$. This suspension was obtained by hydration of lime coming from the lime kiln of our industrial partner.

The reaction begins with the bubbling of the gas containing $30 \%$ of $\mathrm{CO}_{2}$. The temperature of the bulk is maintained at the desired value by the use of the thermo-regulated bath. During most of the time of reaction, the $\mathrm{pH}$ of the liquid phase remains almost constant around 12.7 due to the rapid dissolution of solid calcium hydroxide before falling down at the end of the reaction.

All along the reaction, samples of slurry are collected and then titrated with hydrochloric acid solution $2 \mathrm{M}$. The acid is added to the sample under agitation until the $\mathrm{pH}$ reaches the value of 8 so that the PCC particles are

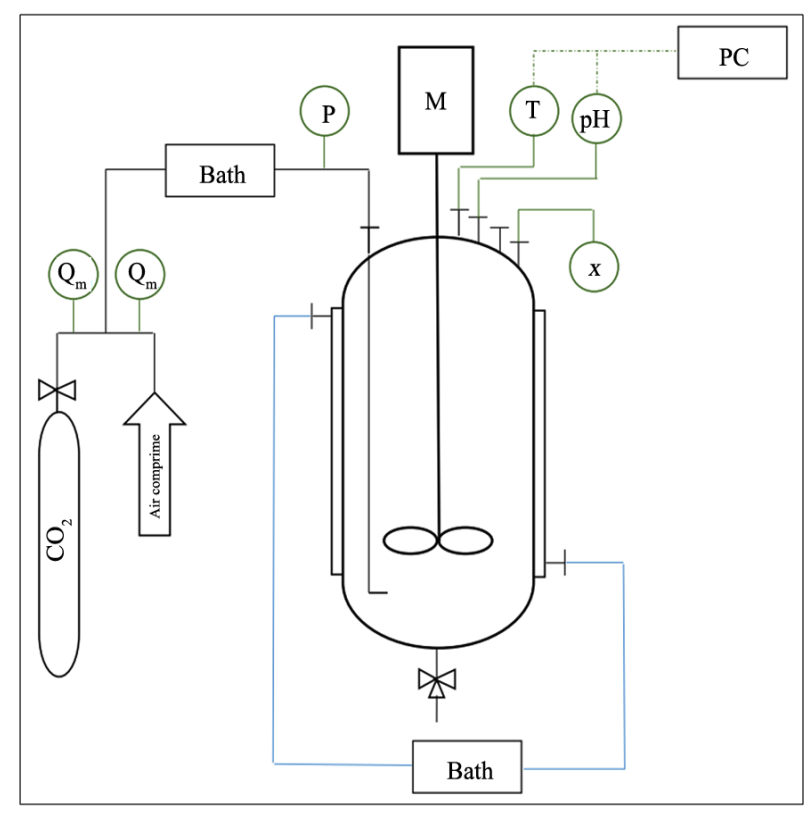

Figure 1. Pilot scheme. 


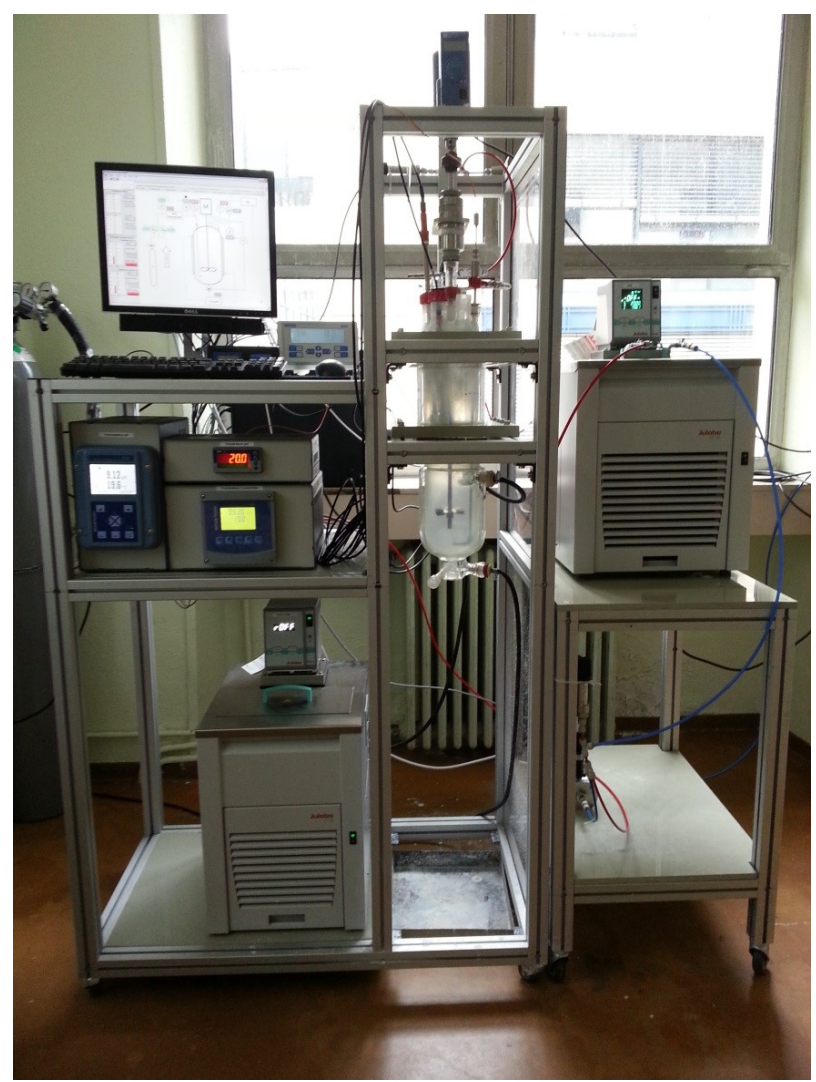

Figure 2. Photograph of the experimental pilot installation.

not dissolved. This operation has two goals: on the one hand to determine the mass of PCC produced and on the other hand to consume the lime, in order to have only PCC remained in the suspension. Each sample is divided in two parts. The first part is analyzed using laser diffraction granulometer (Malvern Mastersizer 2000) in order to get the particle size distribution of the agglomerates and the second part is filtered on Büchner $(0.45 \mu \mathrm{m})$ and dried at $106^{\circ} \mathrm{C}$ during 24 hours to obtain the specific surface area as a function of time using nitrogen adsorption BET method.

The determination of the nucleation and crystal growth rates, which are essential for the modeling of the agglomeration, is achieved thanks to the monitoring of the PCC mass production rate and the specific surface area of the PCC particles. By resolving a population balance in terms of moments, these two values can be determined as a function of time [1].

This work is based on a reference reaction $\left(Q_{\text {gas }}=30 \mathrm{NL} \cdot \mathrm{min}^{-1}, T=21^{\circ} \mathrm{C}\right.$ and $\left.N=500 \mathrm{~min}^{-1}\right)$ and these conditions will then be varied in order to study the influence of the temperature $\left(18^{\circ} \mathrm{C}\right.$ and $\left.24^{\circ} \mathrm{C}\right)$ and the shear rate via the gas flow rate $\left(10\right.$ and $\left.50 \mathrm{NL} \cdot \mathrm{min}^{-1}\right)$ and the agitation speed $\left(300\right.$ and $\left.700 \mathrm{~min}^{-1}\right)$.

\section{Determination of the Agglomeration Kernel}

\subsection{Agglomeration Mechanism}

The agglomeration can be caused by two principal mechanisms: the electrostatic interactions or the crystalline bridges induced by the crystal growth. Thus we tested the carbonation process with a high concentration in sodium chloride $\left(2 \mathrm{~mol} \cdot \mathrm{L}^{-1}\right)$ to check if the modification of the electrical environment will impact the agglomeration. Indeed, with the add of sodium chloride, the ionic strength I goes from $0.07 \mathrm{~mol} \cdot \mathrm{L}^{-1}$ (solubility of calcium hydroxide at $21^{\circ} \mathrm{C}$ is $1.6 \mathrm{~g} \cdot \mathrm{L}^{-1}$ so $0.07 \mathrm{~mol} \cdot \mathrm{L}^{-1}$ ) to $2.07 \mathrm{~mol} \cdot \mathrm{L}^{-1}$.

Nevertheless, we did not observe any significant changes in the properties of the final product regarding either the morphology (Figure 3) or the particle size distribution (Figure 4). It is obviously the crystal growth rate that controls the agglomeration. 

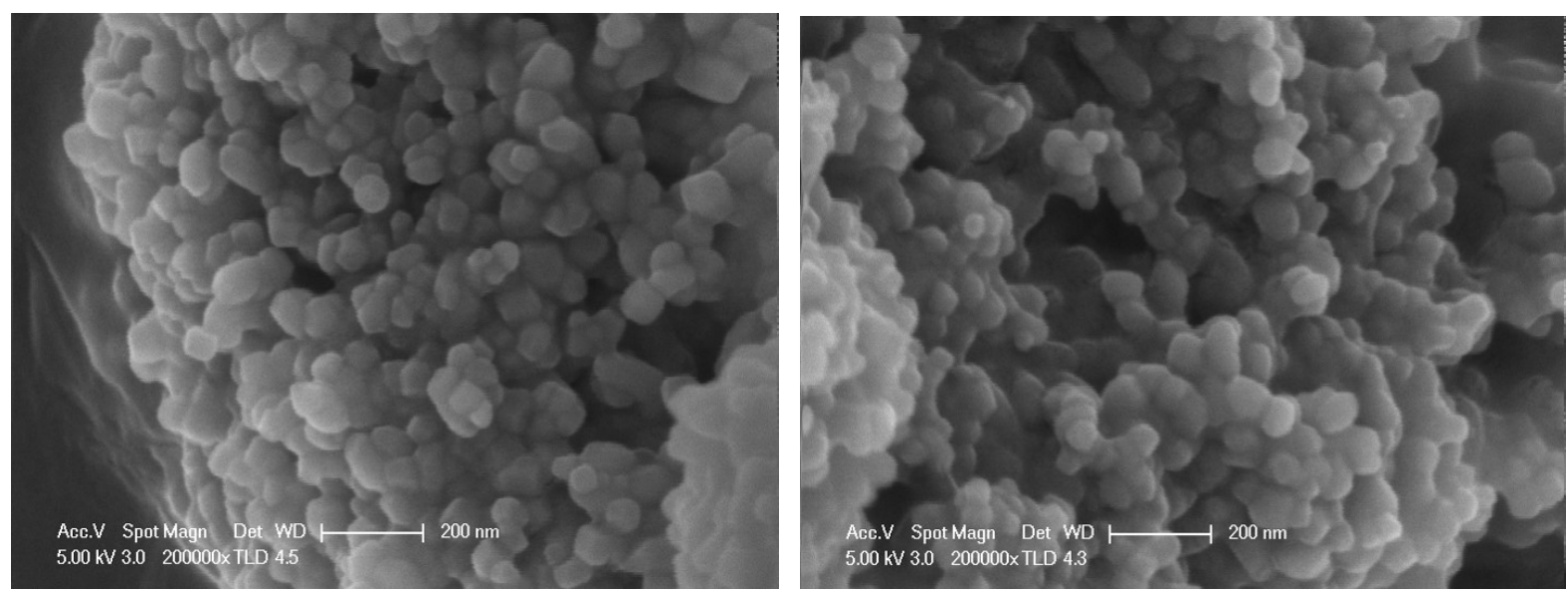

Figure 3. Scanning Electron Microscopy (SEM) image of PCC particles in absence (right) and presence of $\mathrm{NaCl}$ (left).

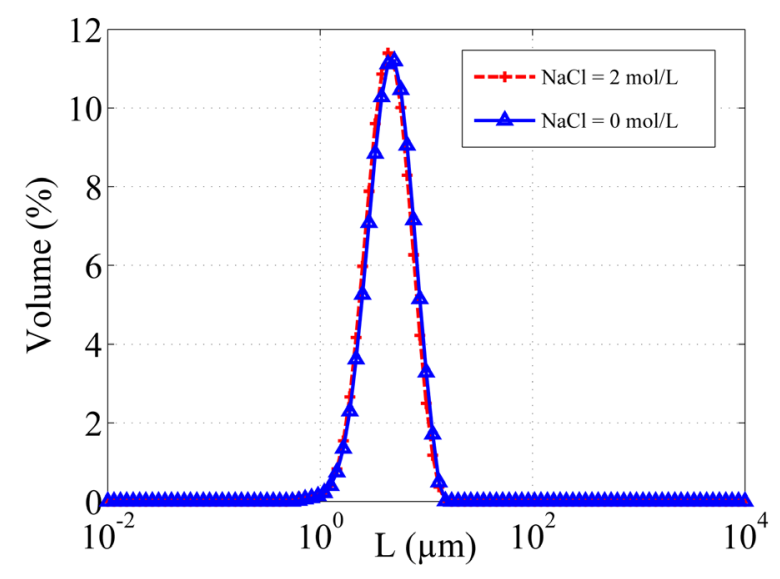

Figure 4. Granulometry of the PCC agglomerates in absence and presence of $\mathrm{NaCl}$.

\subsection{Agglomeration Constant $\beta_{0}$}

The method to determine the agglomeration constant is based on the fitting between the experimental and theoretical values of the moment of order zero from the particle size distribution.

\subsubsection{Experimental Values of the Moment of Order Zero $m_{0}$}

To obtain the experimental values of $m_{0}$ as a function of time, we used the samples collected, which were analyzed with the laser diffraction granulometer.

Indeed, the moments of the particle size distribution are expressed as a function of the number particle size distribution $n(L)$ :

$$
m_{k}=\int_{0}^{\infty} L^{k} n(L) \mathrm{d} L
$$

Yet we can express the number particle size distribution $n(L)$ as a function of the mass particle size distribution $g(L)$ given by the granulometer:

$$
n(L)=\frac{C_{\mathrm{PCC}}}{\phi_{V} \rho_{C}} \frac{g(L)}{L^{3}}
$$

where $C_{\mathrm{PCC}}$ is the mass concentration of PCC in the suspension which varies with time, $\phi_{V}$ is the volume shape factor and $\rho_{C}$ the mass density of the crystals.

Thus when we express the moment of order $\mathrm{k}$ as a function of the data provided by the granulometer, we ob- 
tain:

$$
m_{k}=\frac{C_{\mathrm{PCC}}}{\phi_{V} \rho_{C}} \int_{0}^{\infty} L^{k-3} g(L) \mathrm{d} L
$$

and

$$
m_{0}=\frac{C_{\mathrm{PCC}}}{\phi_{V} \rho_{C}} \int_{0}^{\infty} \frac{g(L)}{L^{3}} \mathrm{~d} L
$$

\subsubsection{Theoretical Values of the Moment of Order Zero $m_{0}$}

The carbonation in presence of $\mathrm{NaCl}$ revealed that the electrostatic interactions were not responsible for the agglomeration so it is obviously the crystal growth by the building of crystalline bridges. Thus, we can test an agglomeration model on the PCC particles in a batch reactor, including terms of nucleation, growth and agglomeration. The Equation (5) details the expression of the agglomeration kernel $\beta$ that includes a constant $\beta_{0}$ and the crystal growth rate $G(t)[17]$ :

$$
\beta\left(\mathrm{m}^{3} \cdot \mathrm{s}^{-1}\right)=\beta_{0} G(t)
$$

The parameter $\beta_{0}$ is an indication of the product tendency to agglomerate. It is a constant that gathers the principal information about the influence of:

- the properties of the substance: shape of the particle (sphere, needle, plate...) or surface state (sticky, rough...)

- hydrodynamics, shear rate...

- the environment in which the substance evolves ( $\mathrm{pH}$, temperature, ionic strength...)

The unit of $\beta_{0}$ depends on the expression of the agglomeration kernel $\beta$. In our case, as the crystal growth rate $G$ (in $\mathrm{m} \cdot \mathrm{s}^{-1}$ ) is included in the expression of $\beta$, the agglomeration constant $\beta_{0}$ will be expressed in $\mathrm{m}^{2}$.

For the moment of zero order, the following expression can be written [26]:

$$
\frac{\mathrm{d} m_{0}}{\mathrm{~d} t}=r_{N}-\frac{1}{2} \beta_{0} G(t) m_{0}^{2}
$$

In the equation above, two necessary values have to be determined in order to access to the agglomeration kernel and constant: the nucleation and the crystal growth rates. In a previous work [1], a method was presented to calculate $r_{N}$ and $G$ by monitoring of two macroscopic parameters: the PCC mass produced and the specific surface area as a function of time. The mathematical treatment provides the expressions of the nucleation and crystal growth rates [1]:

$$
G=\frac{1}{3} \frac{\phi_{S}}{\phi_{V} \rho_{C}} \frac{1}{a_{S}(t) t}=\frac{1}{3} \frac{\phi_{S}}{\phi_{V} \rho_{C}} \frac{1}{z}
$$

and

$$
r_{N}=\frac{9}{2} \frac{\phi_{V}^{2} \rho_{C}^{2}}{\phi_{S}^{3}} r_{M}\left[\left(z^{\prime}\right)^{3}+4 z z^{\prime} z^{\prime \prime}+z^{2} z^{\prime \prime \prime}\right]
$$

where

- $z=a_{s}(t) t$ with $a_{s}(t)$ the PCC monoparticles specific surface area as a function of time;

- $\quad z^{\prime}, z^{\prime \prime}$ and $z^{\prime \prime \prime}$ are the first, second and third derivatives of $z$;

- $r_{M}$ is the PCC mass production rate, which is constant during the carbonation but varies with the reaction conditions (Table 1).

The specific surface area $a_{s}(t)$ is under the form of an empirical expression determined thanks to the the fitting of experimental measurements of the specific surface area during carbonation ( $a_{s}$ in $\mathrm{m}^{2} \mathrm{~kg}^{-1}$ and $t$ in s):

$$
a_{s}(t)=\frac{b}{t^{n}}+c, 0<n<1
$$

The values $b, n, c$ and $r_{M}$ are gathered in Table 1 .

The differential equation of the zero order moment (Equation (6)) has the form of a classical Riccati equation, which has an analytical solution if a particular solution is known. In our case, we could not find a particular 
Table 1. Values of the PCC production rate $r_{M}$ and coefficients $b, n$ and $c$ of Equation (9) for the different reaction conditions.

\begin{tabular}{ccccccc}
\hline $\mathrm{T}$ & $\mathrm{Q}$ & $\mathrm{N}$ & $b$ & $n$ & $c$ & $r_{M}$ \\
\hline${ }^{\circ} \mathrm{C}$ & $\mathrm{NL} \cdot \mathrm{min}^{-1}$ & $\mathrm{~min}^{-1}$ & $\mathrm{~m}^{2} \cdot \mathrm{s}^{\mathrm{n}} \cdot \mathrm{kg}^{-1}$ & - & $\mathrm{m}^{2} \cdot \mathrm{kg}^{-1}$ & $\mathrm{~kg} \cdot \mathrm{m}^{-3} \cdot \mathrm{s}^{-1}$ \\
\hline 18 & 30 & 500 & $3.25 \times 10^{-5}$ & 0.526 & $2.21 \times 10^{4}$ & 0.046 \\
21 & 30 & 300 & $3.91 \times 10^{-5}$ & 0.670 & $1.82 \times 10^{4}$ & 0.024 \\
21 & 30 & 500 & $3.19 \times 10^{-5}$ & 0.605 & $2.33 \times 10^{4}$ & 0.047 \\
21 & 30 & 700 & $2.80 \times 10^{-5}$ & 0.602 & $2.31 \times 10^{4}$ & 0.047 \\
21 & 10 & 500 & $2.91 \times 10^{-5}$ & 0.570 & $2.12 \times 10^{4}$ & 0.047 \\
21 & 50 & 500 & $3.81 \times 10^{-5}$ & 0.631 & $2.49 \times 10^{4}$ & 0.024 \\
\hline
\end{tabular}

solution and that is why we chose the numerical resolution with the Matlab toolbox and more precisely the function ode45. This function allows the resolution of non-stiff differential equations based on an explicit RungeKutta formula.

\subsubsection{Fitting and Determination of the Agglomeration Constant $\beta_{0}$}

The numerical method to determine the value of the agglomeration constant $\beta_{0}$ is divided in several steps (Figure 5). First, we incorporate the expression of $r_{N}$ and $G$ (Equations (7) and (8)) in the differential Equation (6). Then, we propose an initial value of $\beta_{0}$ in order to solve the Equation (6) and obtain the modelized values of $m_{0}$. As the Equation (4) provides us the experimental values of $m_{0}$, we can compare the calculated values of $m_{0}$ with the experimental ones. If the modelized values of $m_{0}$ do not match the experimental values (comparison with least mean square method), the program changes the value of $\beta_{0}$ and run the resolution of the differential equation again until experimental and modelized results match. This optimization is realized thanks to the function gamultiobj of Matlab toolbox, which can carry out simple or multiobjective optimization using genetic algorithm. Therefore, the value of the agglomeration constant $\beta_{0}$ that is retained, is the one which gives the best match between the experimental values of $m_{0}$ given by the granulometer and the calculated values of $m_{0}$ from the theoretical population balance.

Thus for the reference reaction (Figure 6), we get $\beta_{0}=5.29 \times 10^{-4} \mathrm{~m}^{2}$. We repeated the reference reaction three times in order to determine the relative error of $\beta_{0}$ calculation: we obtained an error of $16 \%$ which is correct for the determination of such a value.

The value of $\beta_{0}$ corresponds to the range of value that can be found in publications. Indeed, the value of the agglomeration kernel $\beta$ that are mentioned in articles for different products varies from $10^{-14}$ to $10^{-17}$ (Table 2).

In our case, the agglomeration kernel $\beta$ is the product of two terms: the agglomeration constant $\beta_{0}$ and the crystal growth rate $G$. As the value of $G$ varies between $10^{-12}$ and $10^{-10} \mathrm{~m} \cdot \mathrm{s}^{-1}$ [1], we expected that $\beta_{0}$ would vary between $10^{-5}$ and $10^{-4} \mathrm{~m}^{2}$.

\section{Influence of the Process Parameter on the Agglomeration Kernel}

For this section, the nucleation and growth rates for each experiment were obtained by the method mentioned in the experimental section. Otherwise, we used the same numerical method as for the reference reaction to determine the agglomeration constant $\beta_{0}$.

\subsection{Influence of the Temperature}

To study the impact of the temperature on the agglomeration constant $\beta_{0}$, we tested carbonations with two different temperatures: $18^{\circ} \mathrm{C}$ and $24^{\circ} \mathrm{C}$. These values of temperature correspond to the range of interest of our industrial partner in order to answer to product specifications regarding specific surface area i.e. between 20 and $30 \mathrm{~m}^{2} \cdot \mathrm{g}^{-1}$.

Thus for $T=18^{\circ} \mathrm{C}, \beta_{0}=3.88 \times 10^{-4} \mathrm{~m}^{2}$ and for $T=24^{\circ} \mathrm{C}, \beta_{0}=6.23 \times 10^{-4} \mathrm{~m}^{2}$ as shown in Figure 7 . 
(1)

Expression of the nucleation rate $r_{N}$ and growth rate $G$ are known (Schnebelen et al, 2015)

(2)

Initial condition of the optimization : $\beta_{0, \text { old }}=\beta_{0, \text { init }}$

(3)

Resolution of Eq. 6 with $\beta_{0, \text { new }}=\beta_{0, \text { old }}$

Comparison with values of Eq.4

Values of $m_{0}$ match :

- No $\beta_{0, \text { old }}=\beta_{0, \text { new }}+\varepsilon \beta_{0}$

- Yes : $\beta_{0}=\beta_{0, \text { new }}$

Figure 5. Illustration of the method to determine the value of the agglomeration constant $\beta_{0}$.

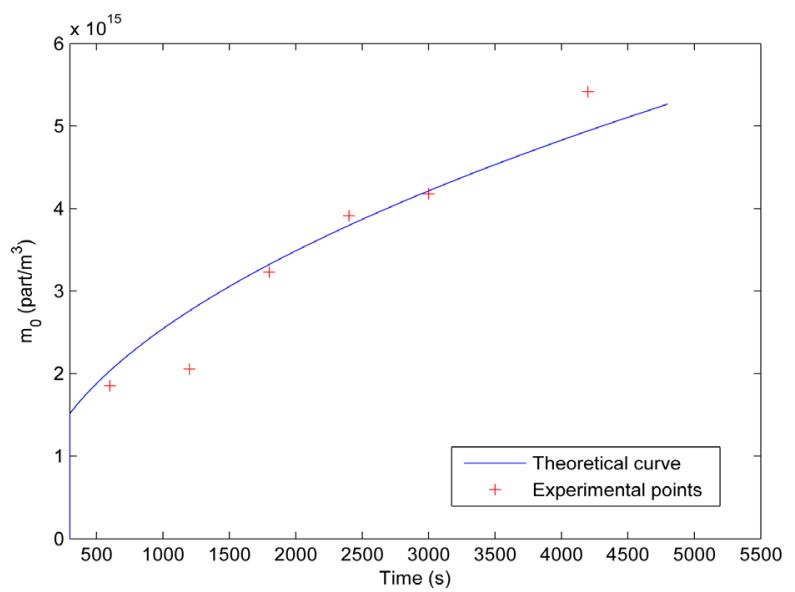

Figure 6. Moment of order zero vs time for the reference reaction.

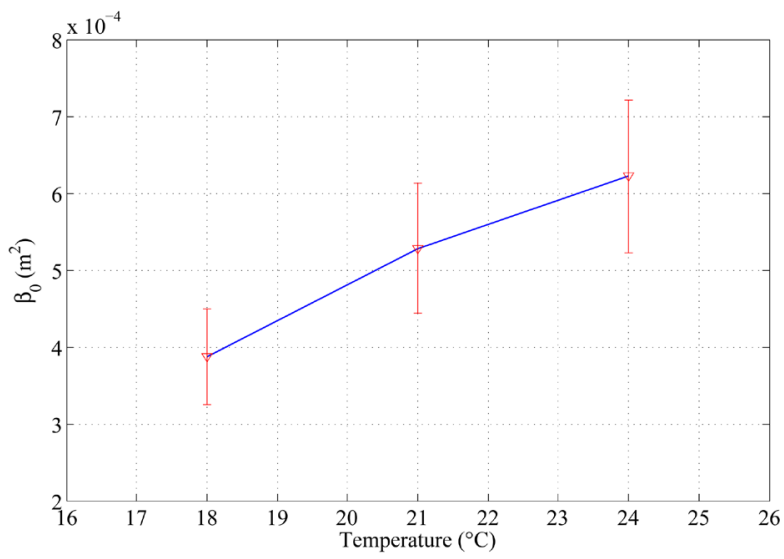

Figure 7. Agglomeration constant $\beta_{0}$ vs temperature.

We can observe that the agglomeration constant increases with the temperature. This phenomenon has already been observed in other works, for example with neodymium oxalate [11] or silica [25].

\subsection{Influence of the Shear Rate}

In the case of agglomeration phenomena, the influence of the shear rate is generally very important. The shear rate is calculated using the following expression: 
Table 2. Values of the agglomeration kernel $\beta$ from diverse authors.

\begin{tabular}{ccc}
\hline Source & Precipitation & Value of $\beta\left(\mathrm{m}^{3 \cdot} \mathrm{s}^{-1}\right)$ \\
\hline Ilievski and White [15] & $\mathrm{Al}(\mathrm{OH})_{3}$ in caustic aluminate solutions & $3 \times 10^{-16}-2.2 \times 10^{-15}$ \\
Collier and Hounslow [17] & Calcite and calcium oxalate monohydrate in solution & $2 \times 10^{-15}-2.10 \times 10^{-14}$ \\
Hollander et al. [23] & Simulation & $5 \times 10^{-16}-1.4 \times 10^{-15}$ \\
Livk and Ilievski [24] & $\mathrm{Al}(\mathrm{OH})_{3}$ in Taylor-Couette and stirred reactor & $10^{-17}-1.4 \times 10^{-15}$ \\
Lallemand et al. [26] & Oxalates in continuous reactor & $8 \times 10^{-15}-2.5 \times 10^{-15}$ \\
\hline
\end{tabular}

$$
\dot{\gamma}=\sqrt{\frac{\varepsilon}{v}}
$$

where $v$ is the cinematic viscosity of the suspension and $\varepsilon$ is the sum of the specific energy injected by mechanical agitation $\varepsilon_{M}$ and the specific energy injected by the gas bubbling $\varepsilon_{G}$ :

$$
\varepsilon\left(\mathrm{W} \cdot \mathrm{kg}^{-1}\right)=\varepsilon_{M}+\varepsilon_{G}
$$

Thus, to study the impact of the shear rate on the agglomeration constant $\beta_{0}$, we modify the agitation speed and the gas flow rate during several trials: $Q_{\text {gas }}=10$ and $50 \mathrm{NL} \cdot \mathrm{min}^{-1}, N=300$ and $700 \mathrm{~min}^{-1}$.

The specific energy injected by gas bubbling is:

$$
\varepsilon_{G}\left(\mathrm{~W} \cdot \mathrm{kg}^{-1}\right)=\frac{P_{G}}{\rho V}=\frac{Q_{\text {gas }} \rho g h}{\rho V}=\frac{v S g h}{S h}=v g
$$

where $v$ is the superficial velocity in $\mathrm{m} \cdot \mathrm{s}^{-1}$;

$P_{G}$ is the power delivered to the system by gas bubbling in $\mathrm{W}$;

$V$ is the suspension volume in $\mathrm{m}^{3}$;

$S$ is the section of the reactor in $\mathrm{m}^{2}$;

$h$ is the height of liquid in the reactor in $\mathrm{m}$.

The energy injected by the mechanical agitation can be calculated by the same formula:

$$
\varepsilon_{M}\left(\mathrm{~W} \cdot \mathrm{kg}^{-1}\right)=\frac{P_{L G}}{\rho V}
$$

During an earlier study on the hydrodynamic conditions of the reactor, the expression of the ratio of the power delivered to the system by mechanical stirring under gas bubbling $P_{L G}$ on the power delivered without gas $P_{L}$ has been determined:

$$
\frac{P_{L G}}{P_{L}}=\exp \left(-2.68 N a^{0.68} \mathrm{Fr}^{0.62}\right)
$$

where $P_{L}=N_{p} N^{3} D_{A}^{5}$ with $N_{p}=1.7$.

This ratio is a function of two dimensionless numbers: the aeration number $N a=\frac{Q_{\text {gas }}}{N D_{A}^{3}}$ and the Froud number $F r=\frac{D_{A} N^{2}}{g}$ where $D_{A}$ is the impeller diameter (in m) and g the gravity acceleration $\left(9.81 \mathrm{~m}^{2} \cdot \mathrm{s}^{-1}\right)$.

Then, it is possible to determine the shear rate for each carbonation process and, by using the same method as for the reference conditions, the value of the agglomeration constant $\beta_{0}$ is determined thanks to the experimental moments of order zero (Figure 8 and Table 3 ).

Thanks to the presented values, we can propose an expression of the agglomeration constant $\beta_{0}$ as a function of the shear rate for $\dot{\gamma}=\left[240-360 \mathrm{~s}^{-1}\right]$ :

$$
\beta_{0}\left(\mathrm{~m}^{2}\right)=-1.17 \times 10^{-7} \dot{\gamma}^{2}+7.17 \times 10^{-5} \dot{\gamma}-1.04 \times 10^{-2}
$$




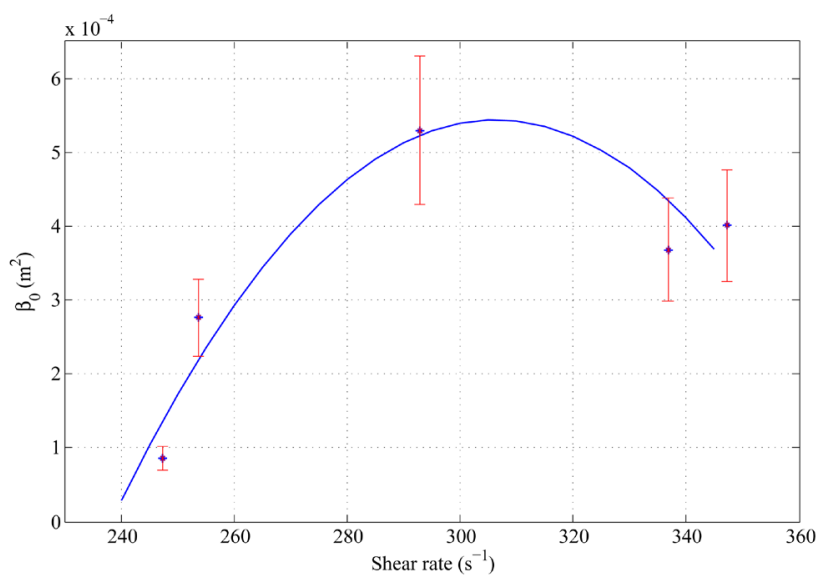

Figure 8. Agglomeration constant $\beta_{0}$ vs shear rate.

Table 3. Values of the agglomeration constant $\beta_{0}$ at different shear rates.

\begin{tabular}{ccccccc}
\hline$\dot{\gamma}\left(\mathrm{s}^{-1}\right)$ & 247 & 254 & 293 & 337 & 347 \\
\hline$\beta_{0} \cdot 10^{4}\left(\mathrm{~m}^{2}\right)$ & 0.86 & 2.76 & 5.29 & 3.68 & 4.01 \\
\hline
\end{tabular}

We can remark that the value of the agglomeration constant decreases whether the shear rate is higher or lower. This phenomenon has already been related in the literature [21]. When the shear rate increases, the relative speed between the particles and thus the collision probability is higher. However, the mechanical constraints on the crystalline bridges are more important leading to the separation of the agglomerated particles. As the agglomeration rate is the product of the collision rate and efficiency, the shear rate has two antagonist effects on the agglomeration and the agglomeration rate seems to reach a maximum. This was also confirmed by [17] and [16].

\section{Conclusion}

The aim of this work was to determine the mechanism and the kinetics of the agglomeration of calcium carbonate as calcite during the carbonation of a suspension of lime under industrial conditions (high solid concentration and intensive turbulence due to mechanical stirring and gas bubbling). Experiments performed with a high concentration in sodium chloride ( $2 \mathrm{M}$ ) show that the modifications of the electrostatic environment do not change the particle size distribution or the morphology of the agglomerates, so the electrostatic interactions are not responsible for the agglomeration. The principal mechanism of agglomeration is the building of crystalline bridges between the elementary particles of calcium carbonate induced by the crystal growth. Thus, thanks to an agglomeration model including the crystal growth, it is possible to determine the value of the agglomeration constant using a mathematical treatment of the experimental particle size distributions. Finally, by varying different process parameters, it also appears that the agglomeration constant increases with temperature whereas there is an optimum for the shear rate. Regarding to this fact, we can also propose an expression of the agglomeration constant $\beta_{0}$ as a function of the shear rate, which can be used to control the agglomeration in the industrial reactor.

\section{Acknowledgements}

Financial support from Solvay Spécialités France is gratefully acknowledged.

\section{References}

[1] Schnebelen, M., Ricaud, M., Jakob, A., Sy, D., Plasari, E. and Muhr, H. (2015) Determination of Crystallization Kinetics and Size Distribution Parameters of Agglomerated Calcium Carbonate Nanoparticles during the Carbonation of a Suspension of Lime. Crystal Structure Theory and Applications, 4, 16-27.

[2] Liao, P. and Hulburt, H. (1976) Agglomeration Process in Suspension Crystallization. Proceedings of 69th Annual Meeting American Institute of Chemical Engineers. 
[3] Tavare, N., Shah, M. and Garside, J. (1985) Crystallization and Agglomeration Kinetics of Nickel Ammonium-Sulfate in MSMPR Crystallizer. Powder Technology, 44, 13-18. http://dx.doi.org/10.1016/0032-5910(85)85015-4

[4] Hounslow, M. (1990) Nucleation, Growth, and Aggregation Rates from Steady-State Experimental-Data. AIChE Journal, 36, 1748-1752. http://dx.doi.org/10.1002/aic.690361117

[5] Hostomsky, J. and Jones, A. (1991) Calcium-Carbonate Crystallization, Agglomeration and Form during Continuous Precipitation from Solution. Journal of Physics D-Applied Physics, 24, 165-170. http://dx.doi.org/10.1088/0022-3727/24/2/012

[6] Rohani, S. and Chen, M. (1993) Aggregation and Precipitation Kinetics of Canola Protein by Isoelectric Precipitation. Canadian Journal of Chemical Engineering, 71, 689-698. http://dx.doi.org/10.1002/cjce.5450710506

[7] Wojcik, J.A. and Jones, A.G. (1997) Experimental Investigation into Dynamics and Stability of Continuous MSMPR Agglomerative Precipitation of $\mathrm{CaCO}_{3}$ Crystals. Chemical Engineering Research \& Design, 75, 113-118. http://dx.doi.org/10.1205/026387697523516

[8] Li, T.S., Livk, I. and Ilievski, D. (2001) The Influence of Crystallizer Configuration on the Accuracy and Precision of Gibbsite Crystallization Kinetics Estimates. Chemical Engineering Science, 56, 2511-2519. http://dx.doi.org/10.1016/S0009-2509(00)00451-6

[9] Georgieva, P., Meireles, M.J. and de Azevedo, S.F. (2003) Knowledge-Based Hybrid Modelling of a Batch Crystallization When Accounting for Nucleation, Growth and Agglomeration Phenomena. Chemical Engineering Science, 58, 3699-3713. http://dx.doi.org/10.1016/S0009-2509(03)00260-4

[10] Zauner, R. and Jones, A.G. (2000) Determination of Nucleation, Growth, Agglomeration and Disruption Kinetics from Experimental Precipitation Data: The Calcium Oxalate System. Chemical Engineering Science, 55, 4219-4232. http://dx.doi.org/10.1016/S0009-2509(00)00059-2

[11] Lallemand, S., Bertrand, M. and Plasari, E. (2012) Physical Simulation of Precipitation of Radioactive Element Oxalates by Using the Harmless Neodymium Oxalate for Studying the Agglomeration Phenomena. Journal of Crystal Growth, 342, 42-49. http://dx.doi.org/10.1016/j.jcrysgro.2011.01.079

[12] Ilievski, D. and Livk, I. (2006) An Agglomeration Efficiency Model for Gibbsite Precipitation in a Turbulently Stirred Vessel. Chemical Engineering Science, 61, 2010-2022. http://dx.doi.org/10.1016/j.ces.2005.10.051

[13] Lindenberg, C., Scholl, J., Vicum, L., Mazotti, M. and Brozio, J. (2008) L-Glutamic Acid Precipitation: Agglomeration Effects. Crystal Growth and Design, 8, 224-237. http://dx.doi.org/10.1021/cg070161f

[14] Hounslow, M.J., Ryall, R.L. and Marshall, V.R. (1988) A Discretized Population Balance for Nucleation, Growth, and Aggregation. AIChE Journal, 34, 1821-1832. http://dx.doi.org/10.1002/aic.690341108

[15] Ilievski, D. and White, E. (1994) Agglomeration during Precipitation: Agglomeration Mechanism Identification for $\mathrm{Al}(\mathrm{OH})_{3}$ Crystals in Stirred Caustic Aluminate Solutions. Chemical Engineering Science, 49, 3227-3239. http://dx.doi.org/10.1016/0009-2509(94)E0060-4

[16] Bramley, A.S., Hounslow, M.J. and Ryall, R.L. (1996) Aggregation during Precipitation from Solution: A Method for Extracting Rates from Experimental Data. Journal of Colloid and Interface Science, 183, 155-165. http://dx.doi.org/10.1006/jcis.1996.0530

[17] Collier, A.P. and Hounslow, M.J. (1999) Growth and Aggregation Rates for Calcite and Calcium Oxalate Monohydrate. AIChE Journal, 45, 2298-2305. http://dx.doi.org/10.1002/aic.690451105

[18] Tourbin, M. and Frances, C. (2008) Experimental Characterization and Population Balance Modelling of the Dense Silica Suspensions Aggregation Process. Chemical Engineering Science, 63, 5239-5251. http://dx.doi.org/10.1016/j.ces.2008.06.028

[19] Lemanowicz, M., Al-Rashed, M.H., Gierczycki, A.T. and Kocurek, J. (2009) Application of the QMOM in Research on the Behavior of Solid-Liquid Suspensions. Chemical and Biochemical Engineering Quarterly, 23, 143-151.

[20] Smoluchowski, M. (1917) Mathematical Theory of the Kinetics of the Coagulation of Colloidal Solutions. Zeitschrift für Physikalische Chemie, 19, 129-135.

[21] Mumtaz, H.S., Hounslow, M.J., Seaton, N.A. and Paterson, W.R. (1997) Orthokinetic Aggregation during Precipitation. A Computational Model for Calcium Oxalate Monohydrate. Chemical Engineering Research and Design, 75, $152-159$.

[22] Hounslow, M., Mumtaz, H., Collier, A., Barrick, J. and Bramley, A. (2001) A Micro-Mechanical Model for the Rate of Aggregation during Precipitation from Solution. Chemical Engineering Science, 56, 2543-2552. http://dx.doi.org/10.1016/S0009-2509(00)00436-X

[23] Hollander, E.D., Derksen, J.J., Bruinsma, O.S.L., van den Akker, H.E.A. and van Rosmalen, G.M. (2001) A Numerical Study on the Coupling of Hydrodynamics and Orthokinetic Agglomeration. Chemical Engineering Science, 56, 25312541. http://dx.doi.org/10.1016/S0009-2509(00)00435-8

[24] Livk, I. and Ilievski, D. (2007) A Macroscopic Agglomeration Kernel Model for Gibbsite Precipitation in Turbulent 
and Laminar Flows. Chemical Engineering Science, 62, 3787-3797. http://dx.doi.org/10.1016/j.ces.2007.03.030

[25] Schaer, E., Ravetti, R. and Plasari, E. (2001) Study of Silica Particles Aggregation in a Batch Agitated Vessel. Chemical Engineering and Processing, 40, 277-293. http://dx.doi.org/10.1016/s0255-2701(00)00124-0

[26] Salvatori, F., Muhr, H. and Plasari, E. (2005) A New Solution for Closure Problem in Crystallization Modeling Using Moments Method. Powder Technology, 157, 27-32. http://dx.doi.org/10.1016/j.powtec.2005.05.008 